\title{
Engineering human seleno-glutaredoxin containing consecutive rare codons as an artificial glutathione peroxidase
}

\author{
ZHANG Wei ${ }^{1}$, LUO Quan ${ }^{1}$, WANG XiaoPing ${ }^{2}$, ZHANG DongMei ${ }^{1}$, MIAO Lu ${ }^{1}$, XU JiaYun ${ }^{1}$, \\ LUO GuiMin ${ }^{2}$, SHEN JiaCong ${ }^{1} \&$ LIU JunQiu ${ }^{*}$ \\ ${ }^{1}$ State Key Laboratory of Supramolecular Structure and Materials, Jilin University, Changchun 130012, China \\ ${ }^{2}$ Key Laboratory for Molecular Enzymology and Engineering of Ministry of Education, Jilin University, Changchun 130012, China
}

Received February 28, 2011; accepted April 5, 2011

\begin{abstract}
The active center of human glutaredoxin (hGrx1) shares a common thioredoxin fold and specific affinity for substrate glutathione (GSH) with natural glutathione peroxidase (GPx). hGrx1 was redesigned to introduce the catalytic selenocysteine residue to imitate the function of antioxidant selenoenzyme GPx in vivo. The human hGrx1 scaffold is a good candidate for potential medical application compared with other animal-originated protein scaffolds. Two consecutive rare codons (AGG-AGG) in the open reading frame of $h G r x 1$ mRNA encoding Arg26-Arg27 residues can reduce seleno-hGrx1 expression level significantly in the Cys auxotrophic Escherichia coli strain BL21cysE51. Therefore, we optimized the rare codons, which resulted in a remarkable increase of the expression level in the Cys auxotrophic cells, which may be sufficient for future medical production. The engineered artificial selenoenzyme displays high GPx catalytic activity, rivaling that of some natural GPx proteins. Kinetic analysis of the engineered seleno-hGrx1 showed a typical ping-pong kinetic mechanism; its catalytic properties are similar to those of some naturally occurring GPx proteins.
\end{abstract}

selenium, selenocysteine, rare codon, artificial enzyme, glutathione peroxidase, glutaredoxin

Citation: Zhang W, Luo Q, Wang X P, et al. Engineering human seleno-glutaredoxin containing consecutive rare codons as an artificial glutathione peroxidase. Chin Sci Bull, 2012, 57: 25-32, doi: 10.1007/s11434-011-4711-9

Oxidative stress is associated with a wide range of human diseases, including atherosclerosis and related vascular diseases, cancer, immunologic disorders, and neurodegenerative diseases, either directly or indirectly. An increase in intracellular concentrations of oxidizing species, such as superoxide radicals, hydroxyl radicals, or hydrogen peroxide $\left(\mathrm{H}_{2} \mathrm{O}_{2}\right)$, leads to the oxidation of proteins, DNA, and membranes, and thus mitochondrial dysfunction and ultimately cell death [1]. Cells contain a large number of antioxidants, including non-enzymatic and enzymatic antioxidant systems to prevent or repair the damage caused by reactive oxygen species, as well as to regulate redox-sensitive signaling pathways [2]. Antioxidant enzymes, including superoxide dismutase, which converts superoxide radicals to $\mathrm{H}_{2} \mathrm{O}_{2}$, and glutathione peroxidase $(\mathrm{GPx})$ and catalase,

*Corresponding author (email: junqiuliu@jlu.edu.cn) which convert $\mathrm{H}_{2} \mathrm{O}_{2}$ to water, cooperate for protection against oxidative stress [3]. Intrinsic disadvantages of natural selenocysteine-containing GPxs have limited their application, and many research efforts have been made to mimic the functions of GPx for potential medical application. For example, the low-molecular-weight GPx mimic 2-phenyl-1,2-benzoisoselenazol-3(2H)-one (Ebselen) has entered phase II clinical trials for the treatment of acute ischemic stroke [4]. However, there has been no report of a human natural protein scaffold that possesses a highly specific GSH-binding site for the design of a novel GPx mimic without immunogenicity for clinical application.

The rational design of novel enzyme functions based on a detailed knowledge of protein structure, function, and catalytic mechanism remains a major challenge and a fundamental goal for enzyme engineering, not only to generate new catalysts, but also to understand the catalytic mecha- 
nisms [5,6]. GPx is a well-known antioxidant selenoenzyme that catalyzes the reduction of lipid hydroperoxides to their corresponding alcohols and the conversion of free $\mathrm{H}_{2} \mathrm{O}_{2}$ to water with reduced glutathione, thus protecting cell components from oxidative damage [7,8]. A great many attempts have been made to redesign GPx because of potential medical importance as an antioxidant [9]. The rational design of a GPX mimic using natural protein scaffolds to introduce catalytic selenium at the active site has attracted considerable interest from biochemists in recent years. Based on the detailed structural and kinetic analysis of the natural GPx and its mutants, it was assumed that the high affinity of GSH toward the catalytic center and the optimum orientation of the sulfhydryl group of GSH to the catalytic selenium group would be critical for constructing a highly efficient GPx for catalyzing the reduction of $\mathrm{H}_{2} \mathrm{O}_{2}$ by GSH [10]. A GSH binding site has been successfully introduced into GPx models using a monoclonal antibody [11] and bioimprinting techniques [12]. The resulting seleniumcontaining proteins exhibited high GPx activity. In addition, some intrinsic GSH-binding sites within the natural protein scaffolds have also been used to incorporate the catalytic selenium group for redesigned GPx mimics [13-16]. Furthermore, glutathione S-transferase (GST), sharing a similar structure and GSH binding site with natural GPx, was redesigned to incorporate functional selenium and tellurium in an auxotrophic expression system. The redesigned artificial enzymes demonstrated a remarkable level of GPx activity $[17,18]$. For this proof-of-principle study, we wondered whether other GSH-binding proteins could be redesigned as a scaffold to incorporate the active selenium at the active center to mimic natural GPx.

Glutaredoxin (Grx, EC 1.8.4.3), a well known enzyme with antioxidant function in vivo has an excellent affinity for the catalytic substrate GSH. Therefore, it could be used as a potential candidate for a protein scaffold to mimic GPX function. Furthermore, the yeast glutaredoxin has been reported to have GPx activity, which increased its resistance to oxidative stress [19]. Bovine GPx was also found to have Grx activity, with nearly identical specificity constant for GSH to natural Grx [20]. Casi et al. [21] prepared seleno-glutaredoxin from Escherichia coli using chemical synthesis in vitro; however, the GPx activity of the seleno-glutaredoxin was four orders of magnitude lower than that of bovine GPx. We previously engineered a seleno-Grx domain from mice, whose activity could rival several natural GPxs [22]. However, the engineered seleno-Grx from mice may induce immune reactions when used as antioxidant for clinical application. Therefore, there is an urgent need to engineer a novel GPx model based on a human protein scaffold.

Human glutaredoxin (hGrx1) shares a common thioredoxin fold and specific affinity for GSH with natural GPx, which makes it a good candidate as a scaffold for potential medical application. As shown in Figure 1, the active center

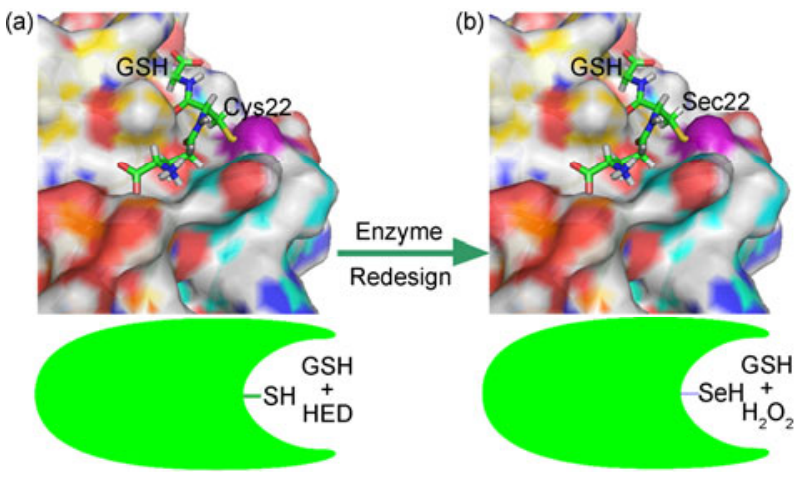

Figure 1 (Color online) Schematic representation of the active site of the genetic engineered seleno-hGrx 1 and the specific recognition of GSH. The structure of the wild-type hGrx1 is derived from the Protein Data Bank (1b4q). (a) The wild-type hGrx1 catalyzes the reduction of HED by GSH. (b) The catalytic center of hGrx1 was redesigned to introduce the catalytic Sec residue at position 22 to mimic GPx activity: the reduction of $\mathrm{H}_{2} \mathrm{O}_{2}$ by GSH.

of hGrx1 was redesigned to introduce the catalytic selenocysteine residue Sec22 for imitating the function of selenoenzyme GPx in vivo. However, the presence of two consecutive AGG-AGG rare codons encoding Arg26-Arg27 significantly decreased the expression of seleno-hGrx1 mRNA in the Cys auxotrophic E. coli strain BL21cysE51. We thus employed a novel strategy for optimizing rare codons (shown in Figure 2), resulting in a remarkable increase in the expression level in Cys auxotrophic cells, which is sufficient for potential future medical production.

\section{Materials and methods}

\subsection{Materials}

The hGrxl gene in plasmid pGEX-T was a generous gift from Prof. Christian Salesse from Laval University, Quebec City, Canada. The mutant hGrx1 (C7S, C25S, C78S, C82S) was kindly donated by Dr. John J. Mieyal from Case Western Reserve University, Cleveland, OH, USA. E. coli strains, including DH5 $\alpha$, BL21(DE3), BL21-CodonPlus-RIL, and the expression vector PET-22b, were purchased from Novagen. The special cysteine auxotrophic E. coli strain BL21cysE51, which was used for introducing the catalytic residue of selenocysteine, was donated by Dr. August Böck from Ludwig-Maximilian University, Munchen, Germany. M9 minimal medium was used as previously described, with minor modifications [23]. Selenocysteine in the M9 production medium was utilized at a concentration of 200 $\mathrm{mg} / \mathrm{L}$. The pX plasmid was prepared from the host $E$. coli strain BL21-CodonPlus-RIL. T4 DNA ligase, Taq polymerase, Pfu polymerase, and Dpn I were from MBI Fermentas, Vilnius, Lithuania. The plasmid Mini-Prep Kit, DNA Gel Extraction Kit, and all the primers for polymerase chain reaction (PCR) were purchased from Sangon, Shanghai, China. The Glutathione Sepharose 4B affinity column and all the other protein purification columns were from GE 

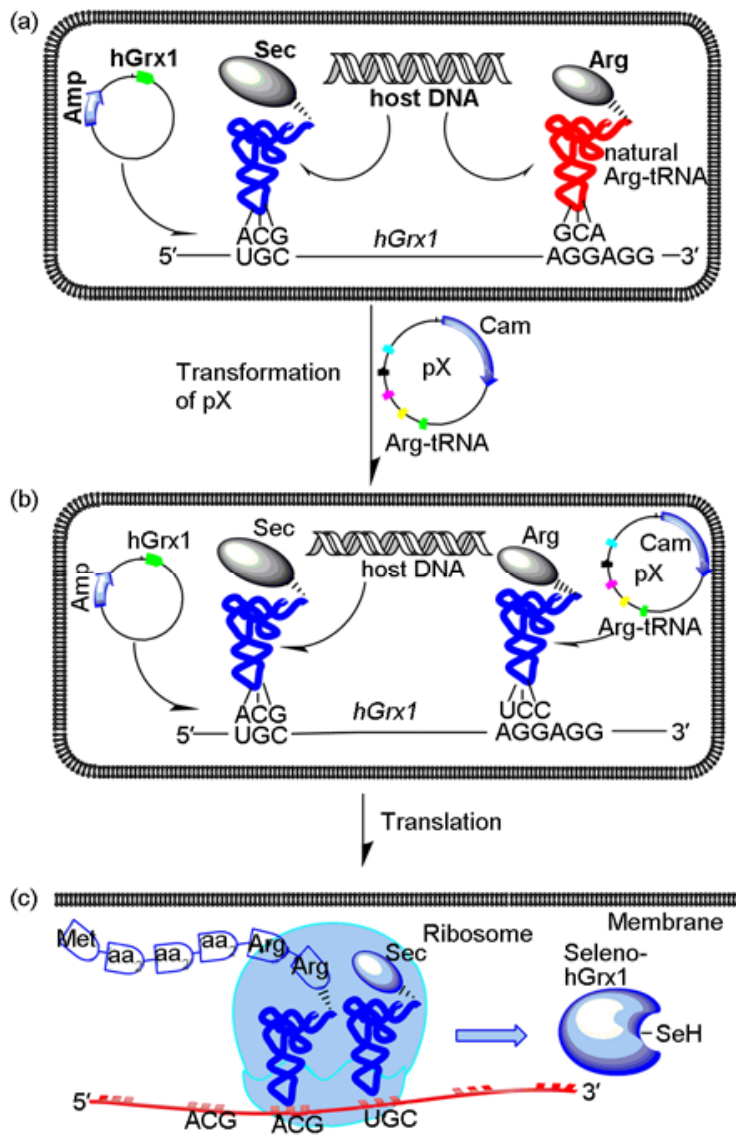

Figure 2 (Color online) Schematic representation of the expression of human seleno-hGrx1 containing consecutive rare codons in the cysteine auxotrophic E. coli strain BL21cysE51. (a) The natural cysteine auxotrophic expression host $E$. coli strain BL21cysE51 does not successfully express seleno-hGrx1 because of intrinsic consecutive rare codons. (b) The transformed plasmid $\mathrm{pX}$ encodes an Arg-tRNA that could match consecutive rare codons in the mRNA of hGrxl well. (c) The translation of seleno-hGrx 1 in the expression host.

Healthcare Life Sciences, Uppsala, Sweden $\beta$-hydroxyethyl disulfide (HED), glutathione reductase (GR), and seleno-L-cysteine were purchased from Sigma-Aldrich, St. Louis, MO, USA. NADPH was from Roche China Co. (Shanghai, China).

\subsection{Construction of expression vector hGrx1-pET-22b}

hGrx1 was cloned into the cloning vector pGEM-T Easy (Promega) and the recombinant plasmid was used as a template for PCR to prepare the hGrx1 gene using the upper primer 5'-GGAATTCGCATATGGCTCAAGAGTTTG-3' and the lower primer 5'-AGTGAAGCTTCTGCAGAGCTCCAAT-3' containing restriction enzymes cleavage sites for Hind III and Nde I, respectively. The pET-22b expression vector and the hGrx1 PCR product were digested with Hind III / Nde I, followed by purification with DNA Gel Extraction Kit (Sangon, Shanghai, China), and then ligated together using T4 DNA ligase at $16^{\circ} \mathrm{C}$ overnight [24]. The recombinant hGrx1-pET-22b was sequenced to check integrity.

\subsection{Overexpression and purification of wild-type hGrx1}

The recombinant hGrx1-pET22b was transformed into $E$. coli strain BL21-CodonPlus-RIL. The expressed hGrx1 was extracted from the bacterial cells, applied to a GSH affinity, and competitively eluted from the column by GSH. The eluate was concentrated and then applied to a $2.6 \mathrm{~cm} \times 80$ cm column of Sephadex G-50 at a flow rate of $0.5 \mathrm{~mL} / \mathrm{min}$ with $0.05(\mathrm{~mol} / \mathrm{L})$ sodium phosphate buffer, $\mathrm{pH} 7.0$ containing $0.15(\mathrm{~mol} / \mathrm{L}) \mathrm{NaCl}$. The salt content was removed using a desalting column Sephadex G-25 column $(1.8 \mathrm{~cm} \times 40 \mathrm{~cm})$. The purity of the sample was verified by SDS-PAGE.

\subsection{Overexpression and purification of seleno-hGrx1}

The recombinant hGrx1-pET22b was transformed into cysteine auxotrophic E. coli strain BL21cysE51. Overexpression of seleno-hGrx1 in the presence of Sec instead of Cys was performed as described for $(\mathrm{Se})_{2}$-thioredoxin, except that the target protein was induced at low temperature of $20^{\circ} \mathrm{C}$ [23]. The protein was also purified by affinity chromatography on Glutathione Sepharose 4B. The protein eluted from the column was further concentrated with Ultrafree-15 centrifugal concentrators (MWCO 3,000, Millipore, Billerica, MA, USA), loaded onto a Sephadex G-50 column, and eluted with $0.05(\mathrm{~mol} / \mathrm{L})$ sodium phosphate buffer, $\mathrm{pH} 7.0$ containing $0.5(\mathrm{~mol} / \mathrm{L}) \mathrm{NaCl}$. The selenohGrx1 fraction was concentrated and loaded to Sephadex G-25 column to remove the salt contents and finally lyophilized. The purity of the sample was verified by SDS-PAGE.

\subsection{Determination of the protein concentration}

Protein concentration was determined by Bradford protein assay (Bio-Rad, Hercules, CA, USA) using bovine serum albumin as the standard [25].

\subsection{Determinations of enzyme activities}

Enzyme activities were measured according to Wilson's method [26]. The reaction was carried out at $37^{\circ} \mathrm{C}$ in $500 \mu \mathrm{L}$ of reaction solution containing potassium phosphate buffer $50 \mathrm{mmol} / \mathrm{L}, \mathrm{pH} 7.0,1.0 \mathrm{mmol} / \mathrm{L} \mathrm{GSH}, 0.4$ units of GR, and $0.5-2.5 \mu \mathrm{g}$ of enzyme. The mixture was preincubated for 5 min, and $50 \mu \mathrm{L}$ of NADPH solution $(2.5 \mathrm{mmol} / \mathrm{L})$ was added. After incubation for another $3 \mathrm{~min}$ at $37^{\circ} \mathrm{C}$, the reaction was initiated by adding $50 \mu \mathrm{L}$ of the second substrate peroxide, such as at a final concentration of $0.5 \mathrm{mmol} / \mathrm{L}$ $\mathrm{H}_{2} \mathrm{O}_{2}, 0.25 \mathrm{mmol} / \mathrm{L}$ cumene hydroperoxide $(\mathrm{CumOOH})$, or $0.25 \mathrm{mmol} / \mathrm{L}$ tert-butyl hydroperoxide $(t-\mathrm{BuOOH})$.

Grx activities of the wild-type and seleno-hGrx1 were determined according to Holgmren's method [27,28]. The reaction was carried out at $25^{\circ} \mathrm{C}$ in $500 \mu \mathrm{L}$ of the reaction mixture containing potassium phosphate buffer $50 \mathrm{mmol} / \mathrm{L}$, 
$\mathrm{pH} 7.0,0.2 \mathrm{mmol} / \mathrm{L} \mathrm{NADPH}, 1 \mathrm{mmol} / \mathrm{L} \mathrm{GSH}, 1 \mathrm{mmol} / \mathrm{L}$ EDTA, 0.4 units GR, $0.5 \mathrm{~mol} / \mathrm{L}$ HED, $0.5-5 \mu \mathrm{g}$ of enzyme.

\subsection{Determination of optimal $\mathrm{pH}$ and temperature for seleno-hGrx1 catalysis}

The initial rates were measured using $1 \mathrm{mmol} / \mathrm{L} \mathrm{GSH}$ and $0.5 \mathrm{mmol} / \mathrm{L} \mathrm{H}_{2} \mathrm{O}_{2}$. The $\mathrm{pH}$ value of the buffer varied from 6.0 to 10.0 to determine the optimal $\mathrm{pH}$ condition for the catalyzed reaction. Similarly, a series of seleno-hGrx1catalyzed reactions were carried out at different temperatures from 25 to $55^{\circ} \mathrm{C}$ to determine the optimal temperature for the reaction.

\subsection{Steady-state kinetics of seleno-hGrx1 catalyzed reaction}

The kinetics analysis of seleno-hGrx1 catalyzed reduction of $\mathrm{H}_{2} \mathrm{O}_{2}$ by $\mathrm{GSH}$ was similar to that of selenium-containing seleno-LuGST1-1 [17]. The initial rates were determined by observing the decrease in NADPH absorption at $340 \mathrm{~nm}$ at a wide range of concentrations of one substrate while the concentration of the other was kept constant. All kinetic experiments were performed in a total volume of $0.5 \mathrm{~mL}$ containing potassium phosphate buffer $50 \mathrm{~mol} / \mathrm{L}$, $\mathrm{pH} 7.0,1$ $\mathrm{mol} / \mathrm{L}$ EDTA, 0.4 unit of GR, $0.25 \mathrm{~mol} / \mathrm{L} \mathrm{NADPH}$, and varying concentrations of $\mathrm{GSH}$ or $\mathrm{H}_{2} \mathrm{O}_{2}$, and seleno-hGrx1. After the preincubation of enzyme with GSH, NADPH, and GR for a few minutes, the reaction was then initiated by the addition of $\mathrm{H}_{2} \mathrm{O}_{2}$. The decrease in absorption without enzyme was recorded and subtracted as background.

\section{Results}

\subsection{Preparation and isolation of seleno-hGrx1}

The preparation method was based on the assumption that an efficient charging of tRNA ${ }^{\mathrm{Cys}}$ with selenocysteine instead of cysteine by cysteinyl-tRNA synthetase occurred when selenocysteine was substituted for cysteine in the culture medium. Thus a selenocysteine residue could replace the cysteine residue in the expressed target protein by the cysteine auxotrophic strain E. coli BL21cysE51 [18,23]. In the case of human glutaredoxin hGrx1, the active residue Cys22 was preserved and then converted to Sec22 during the expression in the host cells BL21cysE51. However, the other four non-specific cysteine residues (Cys7, Cys25, Cys78 and Cys82) would be all substituted by Sec nonspecifically and thus hamper the interpretation of the kinetic performance for the seleno-hGrx 1 if all the five Cys residues were preserved. Therefore, the other four Cys residues, except Cys22, were mutated to Ser residues before introducing selenocysteine at the position Cys22 of hGrx1. The 4 other cysteine residues are not close to the active site and they do not form disulfide bridges; therefore, the substitutions were expected to have no effect on protein folding or catalytic performance [29].

The expression level of seleno-hGrx1 expression in $E$. coli BL21cysE51 increased nearly 5-fold after the rare codons were optimized, compared with that without codon optimization (Figure 3(a)). The production of the artificial selenoenzyme was about $15 \%$ of the total proteins expressed in the cell, as estimated by analysis on SDS-PAGE, both in an active soluble form and as an inactive in inclusion bodies (Figure 3(b)). A high yield of soluble purified protein was achieved at $2 \mathrm{mg} / \mathrm{L}$ of $E$. coli culture for seleno-hGrx1.

\subsection{Peroxidase and Grx activities of seleno-hGrx1}

The GPx and Grx activities of seleno-hGrx1 and other catalysts are listed in Table 1. When HED was used as the substrate, the Grx activity of seleno-hGrx1 (165 U/ $\mu \mathrm{mol})$ was nearly four times lower than that of wild-type hGrx1 (818 $\mathrm{U} / \mu \mathrm{mol})$ under the same conditions. This may be due to the removal of the active Cys22 thiol group, which would involve nucleophilic attack on the glutathionyl disulfide bond forming the Grx-SSG intermediate during the catalytic cycle [30].

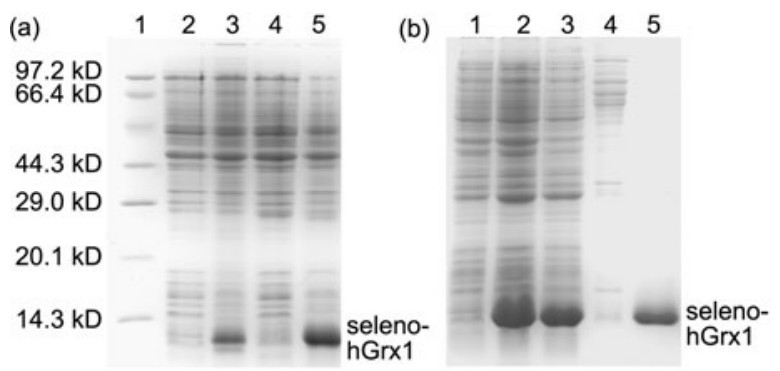

Figure 3 SDS-PAGE of the seleno-hGrx1 during expression and purification. (a) Lane 1, protein molecular weight markers; lanes 2 and 3 are the whole cell extract before and after IPTG induction, respectively, without rare codon optimization; lanes 4 and 5 are the same as lanes 2 and 3 but with rare codon optimization. (b) Lane 1, the whole cell extract without IPTG induction; lane 2, the whole cell extract with IPTG induction; lane 3, supernatant of fragmented cells; lane 4, precipitate of fragmentized cells; lane 5, purified seleno-hGrx1 from the glutathione affinity column.

Table 1 GPx activities of seleno-hGrx1 and other catalysts

\begin{tabular}{ccl}
\hline Catalysts & Substrate & $\begin{array}{c}\text { Activity } \\
(\mathrm{U} / \mu \mathrm{mol})\end{array}$ \\
\hline Wild-type hGrx1 & $\mathrm{H}_{2} \mathrm{O}_{2}$ & 5.2 \\
& $\mathrm{HED}$ & 818 \\
Seleno-hGrx1 & $\mathrm{H}_{2} \mathrm{O}_{2}$ & 136 \\
& $\mathrm{CumOOH}$ & 105 \\
& $t$ - $\mathrm{BuOOH}$ & 121 \\
& $\mathrm{HED}$ & 165 \\
Ebselen [31] & $\mathrm{H}_{2} \mathrm{O}_{2}$ & 0.99 \\
Selenosubtilisin & $\mathrm{H}_{2} \mathrm{O}_{2}$ & 4.6 \\
Seleno-LuGST1-1 [17] & $\mathrm{H}_{2} \mathrm{O}_{2}$ & 2957 \\
Human plasma pGPx [32] & $\mathrm{H}_{2} \mathrm{O}_{2}$ & 302 \\
Rabbit liver GPx [33] & $\mathrm{H}_{2} \mathrm{O}_{2}$ & 5780 \\
\hline
\end{tabular}


The GPx activities of the wild-type hGrx1 and seleno-hGrx1 were estimated by a coupled enzyme system under the same conditions. Wild-type hGrxl showed little GPx activity, whereas seleno-hGrx1 displayed an excellent activity of $136 \mathrm{U} / \mu \mathrm{mol}$ (Table 1 ). The seleno-hGrx1 catalyzed reaction rate was proportional to the concentration of enzyme (Figure 4). The GPx activity of seleno-hGrx1 in catalyzing the reduction of $\mathrm{H}_{2} \mathrm{O}_{2}$ by $\mathrm{GSH}$ could be attributed to the Sec22 residue. Although seleno-hGrx1 was less efficient than some natural GPx for the reduction of $\mathrm{H}_{2} \mathrm{O}_{2}$ by GSH, it was at least 30-fold and 137-fold more efficient than the first semisynthetic GPx mimic selenosubtilisin and the well-known GPx model compound Ebselen [31]. Its activity was even comparable with those of human natural GPx, such as human plasma pGPx whose activity was 302 $\mathrm{U} / \mu \mathrm{mol}[32]$.

\subsection{Optimal pH and temperature for seleno-hGrx1 catalyzed reduction of $\mathrm{H}_{2} \mathrm{O}_{2}$ by GSH}

The GPx activity of seleno-hGrx1 was examined over a wide range of $\mathrm{pH}$ values (from 6.0 to 10.0) and temperatures (from 25.0 to $55.0^{\circ} \mathrm{C}$ ). Figure 5 shows that the optimal $\mathrm{pH}$ of seleno-hGrx1 was 7.6, slightly lower than that of natural GPx (8.8), and the optimal temperature of seleno-hGrx1 was $48^{\circ} \mathrm{C}$, which was near the optimal value for

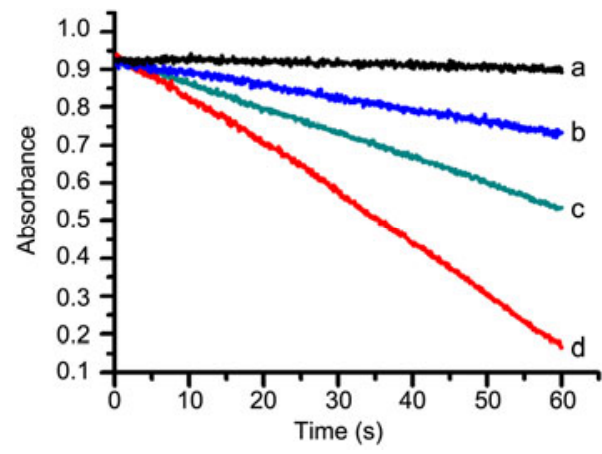

Figure 4 Absorbance vs. time during the catalytic reduction of $\mathrm{H}_{2} \mathrm{O}_{2}$ by GSH monitored at $340 \mathrm{~nm}$ for NADPH decrease with different concentration of enzyme. (a) in the absence of the enzyme sample; (b) $0.07 \mu \mathrm{mol} / \mathrm{L}$; (c) $0.2 \mu \mathrm{mol} / \mathrm{L}$; (d) $0.4 \mu \mathrm{mol} / \mathrm{L}$
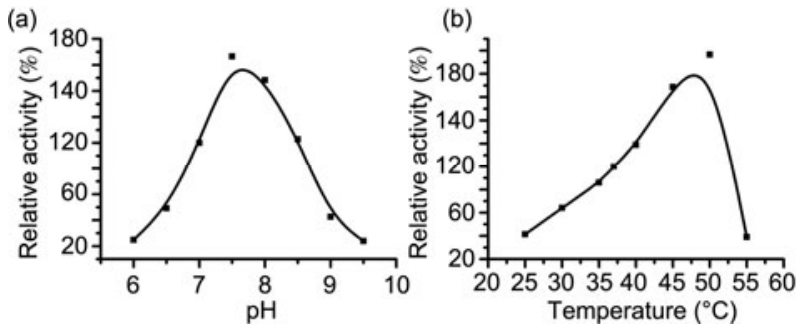

Figure 5 Effects of $\mathrm{pH}$ and temperature on GSH-dependent GPx activity of seleno-hGrx1. (a) Relative GPx activity as a function of pH. (b) Relative GPx activity as a function of temperature. The activity was determined at the concentrations of $1 \mathrm{mmol} / \mathrm{L} \mathrm{GSH}$ and $0.5 \mathrm{mmol} / \mathrm{L} \mathrm{H}_{2} \mathrm{O}_{2}$. The activity detected at $37^{\circ} \mathrm{C}$ and $\mathrm{pH} 7.0$ was defined as $100 \%$. natural GPx $\left(55^{\circ} \mathrm{C}\right)$ [34]. The GPx activities of seleno-hGrx 1 at $37^{\circ} \mathrm{C}$ and $\mathrm{pH} 7.0$ were $56 \%$ and $64 \%$ of their maximums of $243 \mathrm{U} / \mu \mathrm{mol}$ at $48^{\circ} \mathrm{C}$, pH 7.0 and $212 \mathrm{U} / \mu \mathrm{mol}$ at $\mathrm{pH} 7.6,37^{\circ} \mathrm{C}$, respectively.

\subsection{Steady-state kinetics and catalytic mechanism of seleno-hGrx1}

The initial rates for $\mathrm{H}_{2} \mathrm{O}_{2}$ reduction catalyzed by seleno-hGrx1 were determined as a function of substrate concentration at $37.0^{\circ} \mathrm{C}$, pH 7.0 when the concentration of one substrate was varied while the other was fixed. The initial reaction followed Michaelis-Menten kinetics. Double reciprocal plots of the initial rate against substrate concentration revealed the characteristic parallel lines of a ping-pong mechanism, analogous to natural GPx [34] (Figure 6). The enzyme lost GPx activity completely in the presence of GSH and excess iodoacetate, suggesting the presentation of the enzyme-bound selenol in the catalytic cycle [35]. The further characterization of the catalytic mechanism and intermediates need to be fully demonstrated.

\section{Discussion}

Oxidative stress is associated with a wide range of human diseases either directly or indirectly. An increase in the concentrations of oxidizing species would destroy cellular redox balance and lead to the oxidation of proteins, DNA, membrane lipids, and thus mitochondrial dysfunction and ultimately cell death [36]. Anti-oxidative enzymes, such as superoxide dismutase, catalase, and GPx, are the three major reactive oxygen species scavengers in cells and hence protect cells against oxidative damage. The most efficient means of scavenging $\mathrm{H}_{2} \mathrm{O}_{2}$ is mediated by GPx. The intrinsic disadvantages of natural GPx such as difficulty of heterogeneous expression limit its biomedical application. Therefore, much effort has been made to mimic the function of GPx using protein scaffolds and small molecules as hosts to which the catalytic selenium is introduced for therapeutic applications. To date, however, there is no GPx model based on a human protein scaffold with an intrinsic GSH binding site for potential clinical application without immune reactions.

A great many diseases are associated with oxidative stress, forming the basis of antioxidant therapy for these diseases. Ebselen, a synthetic seleno-organic compound, exhibits GPx-like activity of $0.99 \mathrm{U} / \mu \mathrm{mol}$. It has been proven to possess several pharmacodynamic properties, including GPx-like activity and antioxidant activity. It is in phase II clinical trials for the treatment of acute ischemic stroke. Compared with the GPx-like activity of Ebselen, the GPx activity of seleno-hGrx1 is $136 \mathrm{U} / \mu \mathrm{mol}$, about 136 times higher than that of Ebselen. Thus seleno-hGrx1 is should be sufficient as an antioxidant drug, especially as it 

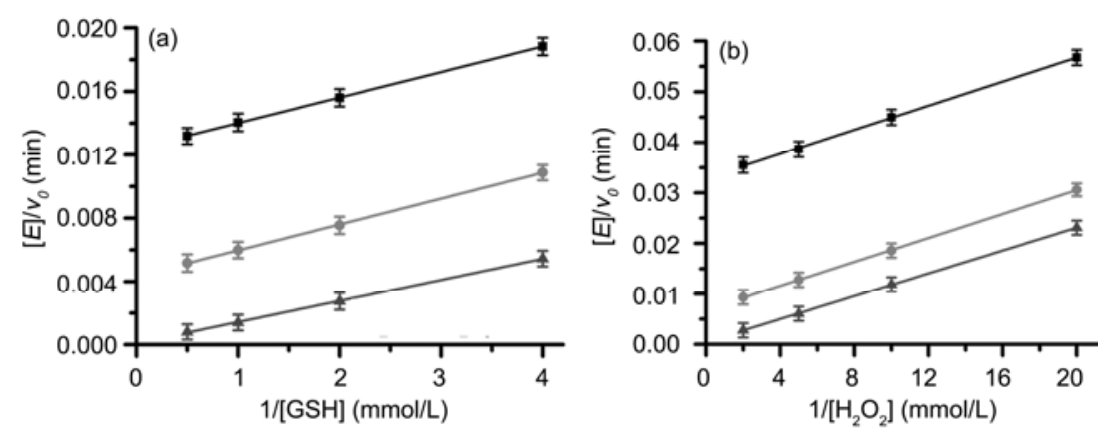

Figure 6 Double-reciprocal plots for the reduction of $\mathrm{H}_{2} \mathrm{O}_{2}$ by GSH catalyzed by seleno-hGrx1. (a) $[E] / v_{0}$ vs. $1 /[\mathrm{GSH}](\mathrm{mmol} / \mathrm{L})$ at $\left[\mathrm{H}_{2} \mathrm{O}_{2}\right]=0.2 \mathrm{mmol} / \mathrm{L}$ $(\bullet), 0.5 \mathrm{mmol} / \mathrm{L}(\bullet)$, and $1 \mathrm{mmol} / \mathrm{L}(\mathbf{\Lambda})$. (b) $[E] / v_{0}$ vs. $1 /\left[\mathrm{H}_{2} \mathrm{O}_{2}\right](\mathrm{mmol} / \mathrm{L})$ at $[\mathrm{GSH}]=0.5 \mathrm{mmol} / \mathrm{L}(\bullet), 1 \mathrm{mmol} / \mathrm{L}(\bullet)$, and $2 \mathrm{mmol} / \mathrm{L}(\mathbf{\Lambda})$.

should have no immunogenicity to humans.

The rational redesign of a novel non-immunogenic enzyme for medical application is a challenge for biochemists and pharmaceutical chemists. To date, few examples of redesigned artificial enzymes that could match the catalytic efficiency of natural enzymes have been observed [37]. Inspired by the successful redesign of seleno-GST [17] and telluro-GST [18], Grx was selected as an excellent scaffold for introducing GPx function because of the overall structural similarity in the GSH-binding domain between Grx and GPx. The seleno-Grx from E. coli did not display high GPx activity when the functional selenium was chemically introduced at the GSH binding site of Grx to simulate GPx activity [21]. By contrast, engineering the Grx domain of mouse thioredoxin/glutaredoxin reductase by substituting Cys with Sec at the active site of Grx presented excellent catalytic performance. However, the immunogenicity and safety to humans of seleno-Grx from mouse limited its further therapeutic application as an antioxidant. Therefore, it is of great importance to explore human protein scaffolds that mimic GPx function for clinical applications.

Human Grx1 is a $12 \mathrm{kD}$ thiol-disulfide oxidoreductase that appears to have a critical role in maintaining the redox environment of the cell and thus protects the organism from oxidative damage. Previous studies have demonstrated that yeast Grx, mouse Grx2, and human mitochondrial Grx2 exhibited GPx activity [19,38]. Furthermore, synthetic selenium-containing Grx3 analogs have been reported to be highly reducing oxidoreductases with enhanced catalytic efficiency [39]. Considering all these previous studies, and the overall structural similarity between GPx and Grx, the evolutionarily related enzyme hGrx1 should provide an excellent potential protein scaffold.

To convert wild-type hGrx 1 into a selenium-containing enzyme, the catalytic Sec residue was incorporated into the GSH-binding site of hGrx1 by substituting the active site Cys22 with Sec22 using Cys auxotrophic E. coli strain BL21cysE51. To avoid the conversion of the other four non-active Cys residues to Secs, they were mutated to Ser residues before expression in the Cys auxotrophic cells
(Figure 1). The four Cys quadruple mutant of hGrx1 (C7S, $\mathrm{C} 25 \mathrm{~S}, \mathrm{C} 78 \mathrm{~S}$, and C82S) displayed the same catalytic efficiency $\left(V_{\max } / K_{\mathrm{M}}\right)$ and specificity for glutathionyl mixed disulfide substrate as that of the wild-type hGrx1 [29]. The quadruple mutant was transformed into the Cys auxotrophic $E$. coli strain and expressed, leading to the specific conversion of the active site Cys22 to the catalytic Sec22.

The presence of two consecutive AGG-AGG rare codons for Arg26-Arg27 residues in the reading frame reduced the seleno-hGrx1 expression level significantly in the Cys auxotrophic E. coli strain BL21cysE51 [40]. Therefore, a novel strategy for optimizing the rare codons was devised for the Cys auxotrophic E. coli strain (Figure 2). The Cys auxotrophic E. coli strain was transformed with plasmid pX encoding an Arg-tRNA that could recognize the rare codons in the mRNA of hGrx1. This strategy resulted in a remarkable increase in the expression level of seleno-hGrx1 in the Cys auxotrophic cells (Figure 3). The expression of the seleno-hGrx1 was induced at a low temperature of $20^{\circ} \mathrm{C}$ to increase its active soluble fraction and decrease the formation of inactive inclusion bodies. The specific affinity of seleno-hGrx 1 for GSH was used to purify the target protein by GSH affinity chromatography.

The engineered seleno-hGrx1 displayed GPx-like peroxidase activity (Table 1). The GPx activity of the engineered seleno-hGrx1 could be mainly attributed to the presence of a more active selenol group compared with the activity of the thiol group. Organic selenium compounds are more reactive as nucleophiles than their sulfur counterparts, as the $\mathrm{p} K \mathrm{a}$ of the selenol group (5.7 in free Sec) is three $\mathrm{pKa}$ units lower than that of the thiol group ( 8.5 in free Cys). Both free Cys and Sec were expected to be $1 \mathrm{pKa}$ unit higher within a polypeptide chain [41]. Seleno-hGrx1 showed a 26-fold increase in GPx activity over the wild-type hGrx1. Modification of the active center of the Sec46Cys mutant of phospholipids GPx was 1000-fold less active than the wild-type enzyme [42]. Therefore, selenium in Sec residue acted as a strong nucleophile and leaving group in the catalytic cycle, indicating that the enhanced GPx activity was mainly attributed to the substitution of the catalytic Cys22 
by a more reactive $\mathrm{Sec}$ residue.

The GPx activity of seleno-hGrx1 was about 22-fold lower than that of the engineered seleno-LuGST-1 we prepared previously [17]. However, it was still close to some natural GPxs, such as human plasma pGPx. This indicated that the GPx catalytic activity depended on the catalytic Sec residue and on some other residues such as the catalytic triad in the active site.

Previous studies have proposed that Grx and GPx share a common "GSH-binding protein" ancestor, based on the similarities in their overall structures and the arrangement of their catalytic residues, despite their low sequence identity and functional differences $[43,44]$. Engineering hGrx1 into an efficient GPx mimic by the conversion of the catalytic Cys22 at the GSH-binding site to Sec22 provides additional proof for the previous assumption that their active-site residues have evolved independently from their common thioredoxin-like ancestor to accommodate different functions and perform various activities in organisms $[45,46]$.

In conclusion, we successfully redesigned and prepared human seleno-hGrx1 by substituting the catalytic residue Cys22 with Sec22 using the Cys auxotrophic expression system, where the four other Cys residues were mutated to Ser residues. The expression level of seleno-hGrx1 in cysteine auxotrophic cells increased five-fold after rare codon optimization. Based on the distinct structural similarities in the specific GSH binding site and geometric orientation of the catalytic Sec residue between seleno-hGrx1 and naturally occurring GPx, the engineered seleno-hGrx1 demonstrated excellent catalytic efficiency. However, its GPx activity was an order of magnitude lower than some of naturally occurring GPx. The engineered seleno-hGrx 1 provides a suitable enzymatic model for further understanding of the relationship between the structure and function of GPx. In addition, the novel redesigned seleno-hGrx1 from a human protein scaffold will be a potential candidate as an antioxidant for medical applications because of its low molecular weight and lack of immunogenicity for humans.

This work was supported by the National Natural Science Foundation of China (91027023, 20874036, 20921003, and 21004028), the Natural Science Foundation for the Youth (21004028), the Natural Science Foundation of China for Outstanding Younger Scientist (20725415), the 111 project (B06009), and the National Basic Research Program (2007CB808006). We also thank Prof. Christian Salesse and Prof. John J. Mieyal for their generous donation of the wild-type hGrxl gene and the mutant gene, respectively.

1 Castro L, Freeman B A. Reactive oxygen species in human health and disease. Nutrition, 2001, 17: 161-165

2 Weydert C J, Cullen J J. Measurement of superoxide dismutase, catalase and glutathione peroxidase in cultured cells and tissue. Nat Protocols, 2010, 5: 51-66

3 Halliwell B. Antioxidants in human health and disease. Annu Rev Nutr, 1996, 16: 33-50

4 Akira O, Haruhiko K, Keiji S, et al. Ebselen in acute middle cerebral artery occlusion: A placebo-controlled, double-blind clinical trial. Cerebrovasc Dis, 1999, 9: 112-118
5 Toscano M D, Woycechowsky K J, Hilvert D. Minimalist active-site redesign: Teaching old enzymes new tricks. Angew Chem Int Ed, 2007, 46: 3212-3236

6 Jochens H, Stiba K, Savile C, et al. Converting an esterase into an epoxide hydrolase. Angew Chem Int Ed, 2009, 48: 3532-3535

7 Yin Y Z, Wang L, Zhang W, et al. New strategies for the design of artificial selenoenzymes (in Chinese). Sci Sin Chim, 2011, 41: 205215

8 Li J, Liu X M, Ji Y T, et al. Biosynthesis of selenosubtilisin: A novel way to target selenium into the active site of subtilisin. Chin Sci Bull, 2008, 53: 2454-2461

9 Huang X, Liu X M, Luo Q, et al. Artificial selenoenzymes: Designed and redesigned. Chem Soc Rev, 2011, 40: 1171-1184

10 Epp O, Ladenstein R, Wendel A. The refined structure of the selenoenzyme glutathione peroxidase at 0.2-nm resolution. Eur J Biochem, 1983, 133: 51-69

11 Ding L, Liu Z, Zhu Z Q, et al. Biochemical characterization of selenium-containing catalytic antibody as a cytosolic glutathione peroxidase mimic. Biochem J, 1998, 332: 251-255

12 Liu J Q, Luo G M, Gao S J, et al. Generation of a glutathione peroxidase-like mimic using bioimprinting and chemical mutation. Chem Commun, 1999, 2: 199-200

13 Jiang Z H, Arnér E S J, Mu Y, et al. Expression of selenocysteine-containing glutathione S-transferase in Escherichia coli. Biochem Biophys Res Commun, 2004, 321: 94-101

14 Zhang K, Zang T Z, Yang W, et al. Single chain antibody displays glutathione S-transferase activity. J Biol Chem, 2006, 281: 1251612520

15 Ren X J, Jemth P, Board P G, et al. A semisynthetic glutathione peroxidase with high catalytic efficiency: Selenoglutathione transferase. Chem Biol, 2002, 9: 789-794

16 Su D, Ren X J, You D L, et al. Generation of three selenium-containing catalytic antibodies with high catalytic efficiency using a novel hapten design method. Arch Biochem Biophys, 2001, 395: 177-184

17 Yu H J, Liu J Q, Böck A, et al. Engineering glutathione transferase to a novel glutathione peroxidase mimic with high catalytic efficiency. $\mathrm{J}$ Biol Chem, 2005, 280: 11930-11935

18 Liu X M, Silks L A, Liu C P, et al. Incorporation of tellurocysteine into glutathione transferase generates high glutathione peroxidase efficiency. Angew Chem Int Ed, 2009, 48: 2020-2023

19 Collinson E J, Wheeler G L, Garrido E O, et al. The yeast glutaredoxins are active as glutathione peroxidases. J Biol Chem, 2002, 277: $16712-16717$

20 Washburn M P, Wells W W. Identification of the dehydroascorbic acid reductase and thioltransferase (glutaredoxin) activities of bovine erythrocyte glutathione peroxidase. Biochem Biophys Res Commun, 1999, 257: 567-571

21 Casi G, Roelfes G, Hilvert D. Selenoglutaredoxin as a glutathione peroxidase mimic. Chembiochem, 2008, 9: 1623-1631

22 Ge Y, Qi Z H, Wang Y, et al. Engineered selenium-containing glutaredoxin displays strong glutathione peroxidase activity rivaling natural enzyme. Int J Biochem Cell B, 2009, 41: 900-906

23 Mueller S, Senn H, Gsell B, et al. The formation of diselenide bridges in proteins by incorporation of selenocysteine residues: Biosynthesis and characterization of (Se)2-thioredoxin. Biochemistry, 1994, 33: 3404-3412

24 Sambrook J, Russell D. Molecular Cloning: A Laboratory Manual. 3rd ed. Cold Spring Harbor: Cold Spring Harbor Laboratory Press, 1989

25 Bradford M M. A rapid and sensitive method for the quantitation of microgram quantities of protein utilizing the principle of protein-dye binding. Anal Biochem, 1976, 72: 248-254

26 Wilson S R, Zucker P A, Huang R R C, et al. Development of synthetic compounds with glutathione peroxidase activity. J Am Chem Soc, 1989, 111: 5936-5939

27 Holmgren A. Glutathione-dependent synthesis of deoxyribonucleotides. Characterization of the enzymatic mechanism of Escherichia coli glutaredoxin. J Biol Chem, 1979, 254: 3672-3678 
28 Lillig C H, Berndt C, Holmgren A. Glutaredoxin systems. Biochim Biophys Acta (BBA)-Gen Subjects, 2008, 1780: 1304-1317

29 Yang Y, Jao S S, Nanduri S, et al. Reactivity of the human thioltransferase (glutaredoxin) C7S, C25S, C78S, C82S mutant and NMR solution structure of its glutathionyl mixed disulfide intermediate reflect catalytic specificity. Biochemistry, 1998, 37: 17145-17156

30 Jao S C, English Ospina S M, Berdis A J, et al. Computational and mutational analysis of human glutaredoxin (thioltransferase): Probing the molecular basis of the low pKa of cysteine 22 and its role in catalysis. Biochemistry, 2006, 45: 4785-4796

31 Mugesh G, Singh H B. Synthetic organoselenium compounds as antioxidants: Glutathione peroxidase activity. Chem Soc Rev, 2000, 29: 347-357

32 Chu F F, Doroshow J H, Esworthy R S. Expression, characterization, and tissue distribution of a new cellular selenium-dependent glutathione peroxidase, GSHPx-GI. J Biol Chem, 1993, 268: 2571-2576

33 Mannervik B. Glutathione peroxidase. Methods Enzymol, 1985, 113: 490-495

34 Flohé L, Günzler WA. Assays of glutathione peroxidase. Methods Enzymol, 1984, 105:114-121

35 Forstrom J W, Zakowski J J, Tappel A L. Identification of the catalytic site of rat liver glutathione peroxidase as selenocysteine. Biochemistry, 1978, 17: 2639-2644

36 Giles G I, Fry F H, Tasker K M, et al. Evaluation of sulfur, selenium and tellurium catalysts with antioxidant potential. Org Biomol Chem, 2003, 1: 4317-4322

37 Wentworth P. Antibody design by man and nature. Science, 2002,
296: $2247-2249$

38 Fernando M R, Lechner J M, Löfgren S, et al. Mitochondrial thioltransferase (glutaredoxin 2) has GSH-dependent and thioredoxin reductase-dependent peroxidase activities in vitro and in lens epithelial cells. FASEB J, 2006, 20: 2645-2647

39 Metanis N, Keinan E, Dawson P E. Synthetic seleno-glutaredoxin 3 analogues are highly reducing oxidoreductases with enhanced catalytic efficiency. J Am Chem Soc, 2006, 128: 16684-16691

40 Spanjaard R A, van Duin J. Translation of the sequence AGG-AGG yields 50\% ribosomal frameshift. Proc Natl Acad Sci USA, 1988, 85: 7967-7971

41 Rabenstein D L, Arnold A P, Guy R D. ${ }^{1}$ H-NMR study of the removal of methylmercury from intact erythrocytes by sulfhydryl compounds. J Inorg Biochem, 1986, 28: 279-287

42 Maiorino M A K, Brigelius-Flohé R, Doria D, et al. Probing the presumed catalytic triad of selenium-containing peroxidases by mutational analysis of phospholipid hydroperoxide glutathione peroxidase (PHGPx). Biol Chem Hoppe Seyler, 1995, 376: 651-660

43 Armstrong R N. Structure, catalytic mechanism, and evolution of the glutathione transferases. Chem Res Toxicol, 1997, 10: 2-18

44 Martin J L. Thioredoxin -a fold for all reasons. Structure, 1995, 3: 245-250

45 Babbitt P C, Gerlt J A. Understanding enzyme superfamilies. J Biol Chem, 1997, 272: 30591-30594

46 Mannervik B, Cameron A D, Fernandez E, et al. An evolutionary approach to the design of glutathione-linked enzymes. Chem Biol Interact, 1998, 111-112: 15-21

Open Access This article is distributed under the terms of the Creative Commons Attribution License which permits any use, distribution, and reproduction in any medium, provided the original author(s) and source are credited. 